\title{
Radial glomerular count- An assessment of human nephrogenesis
}

\author{
Suvarna A. Gulanikar ${ }^{1, *}$, Savita Kadam², SmitaShinde ${ }^{3}$, G. A. Shroff ${ }^{4}$ \\ ${ }^{1,3}$ Assistant Professor, ${ }^{2}$ Associate Professor, ${ }^{4}$ Professor, Dept. of Anatomy, MGM Medical College, Aurangabad, Maharashtra, \\ India
}

*Corresponding Author:

Email: suvarnagulanikar@gmail.com

Received: $30^{\text {th }}$ October, 2017

Accepted: $06^{\text {th }}$ February, 2018

\begin{abstract}
Introduction: The important reason for progression of various glomerulopathies is variable Radial glomerular count [RGC]. There are few studies on the radial glomerular count. Therefore, we conduct this study, with an aim to contribute the knowledge of the human developing kidney by counting radial glomerular number.

Materials and Methods: Thirty normal human fetuses of midgestational period [14 to 28 weeks] were selected for the study. The fetus should not have any congenital anomaly. The fetal kidney tissue slides were prepared and stained in hematoxylin and eosin. Then numbers of glomerular generations were counted and correlate the data with the different gestational groups.

Results: The results showed that the average RGC is gradually increasing from 4 to 15 counts in mid gestational age. The results suggest significant statistical correlation between the period of gestation and the number of glomerular generations (Correlation coefficient value $R^{2}=0.99$ ). The fetal kidney weight and the number of glomerular generations in respective gestational age also shows definite statistical correlations.

Conclusion: The present study concluded that gradually increasing average RGC can express human fetal kidney growth in intrauterine life. The histomorphologic assessment of RGC can be used as relatively better method of estimating gestational age. The observed growth tendencies of fetal glomerulus in human fetal life by RGC could be useful to assess the kidney maturity in the future.
\end{abstract}

Keywords: Glomerulopathies, RGC.

\section{Introduction}

The human kidney develops through a complex process termed 'branching morphogenesis. The initial glomeruli develop at the site of the final bifid branching, located in the most superficial renal cortex near the medullary rays. The other nephrons develop through the poorly understood process of arcade formation and late-phase branching between 22 and 36 weeks gestation. This creates a radial glomerular pattern. It's counting helps to estimate the gestational age. Approximately one generation of new nephrons forms every 2 weeks during the last trimester, ultimately reaching 10-12 generations by term birth., ${ }^{1,2}$

The important reason for progression of various glomerulopathies is variable Radial glomerular count [RGC] .There are few studies on the radial glomerular count. Our study was conducted, with an aim to estimate an average radial glomerular count in cortical zones of the human fetal kidney during 14th to the 28th weeks of gestation, to define gestational age and correlate it with fetal kidney weight.

Shimda K. observed that" the RGC increased steadily with gestational weeks, and gradually reached a plateau of around 12 counts at about 36 weeks. It was not influenced by the intrauterine fetal growth". ${ }^{3}$ Tsuda (1943) stated that the first glomerulus appears when the embryo is 3 inches long. At 4-5 months [13-20 weeks], he found 3-5 layers of glomeruli; at 7-9 months (25-36 weeks) 9-12 layers, and at the 10th month (37- 40 weeks) 10-15 layers. ${ }^{4}$

\section{Materials and Methods}

Thirty human fetal kidneys were selected. After taking written permission of parents, aborted fetuses of midgestational age without any congenital anomaly were collected from the Department of Obstetrics and Gynaecology MGM medical college, Aurangabad. Each specimen fetus was tagged with an identification number.

The proper fixation of fetus was done with $10 \%$ formalin solution for 2 to 3 days. Dissection was done to extract fetal kidneys.

Then these kidneys were undergone tissue processing for dehydration, clearing, embedding. Paraffin blocks were prepared. Tissue blocks were serially sectioned, so each section contains full thickness of cortex and medulla. $7 \mu \mathrm{m}$ thick sections were taken using a rotary microtome. Then prepared tissue slides was stained with Haematoxylin- Eosin (H\& E) stain. All specimens, divided into 8 groups according to gestational age of the fetus for the purpose of study of glomerular generations.

Group 1 - 14 weeks

Group 2 - 16 weeks

Group 3 - 18 weeks

Group 4 - 20 weeks 
Group 5 - 22 weeks

Group 6 - 24 weeks

Group 7 - 26 weeks

Group 8 - 28 weeks

\section{Method to count glomerular generations}

Under the microscope in $4 \mathrm{x}$ magnification radial glomerular count $[\mathrm{RGC}]$ was done. The rows which show clear and maximum number of glomerulus were considered for the study. RGC was calculated by drawing an imaginary straight line from deepest glomerulus at the corticomedullary junction to the most superficial one under the capsule in the middle area between upper and lower poles. Glomerular generations counted between these two points on that straight imaginary line.

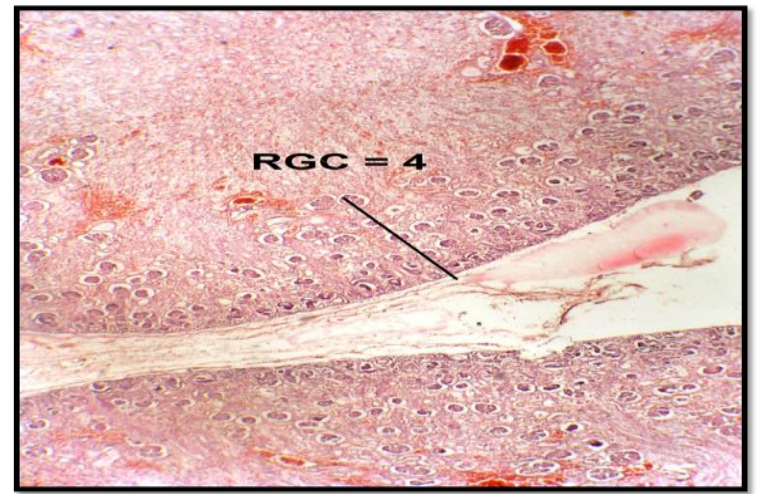

Fig. 1: Shows the slide of 14 weeks fetal kidney with radial glomerular count of four

For each slide five RGC were counted at different regions of cortex. Such two slides were studied per gestational age group. Average RGC is calculated for each age group. Relations with gestational age and average kidney weight were studied.

\section{Statistical analysis}

Regression analysis was used for the correlation between the period of gestation (in weeks) and the number of glomerular generations and the correlation coefficient value was estimated.

\section{Results}

Table 1: Relation of Average Radial glomerular count [RGC] with gestational age of the fetus

\begin{tabular}{|c|c|}
\hline $\begin{array}{c}\text { Gestational Age } \\
\text { [In weeks] }\end{array}$ & $\begin{array}{c}\text { Average RGC } \\
\text { [In number] }\end{array}$ \\
\hline 14 & 4.0 \\
\hline 16 & 5.25 \\
\hline 18 & 6.75 \\
\hline 20 & 9.0 \\
\hline 22 & 10.0 \\
\hline 24 & 11.25 \\
\hline
\end{tabular}

\begin{tabular}{|c|c|}
\hline 26 & 13.0 \\
\hline 28 & 14.75 \\
\hline
\end{tabular}

Graph 1: Average Radial glomerular count (RGC) and gestational age of fetus

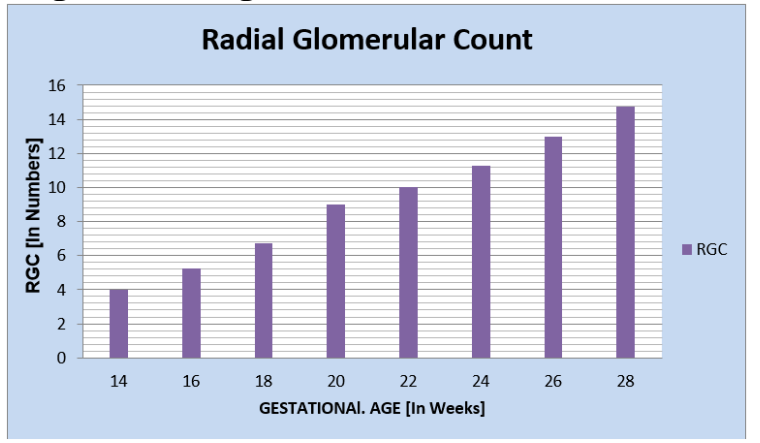

Graph 2: Relation between gestational age and RGC

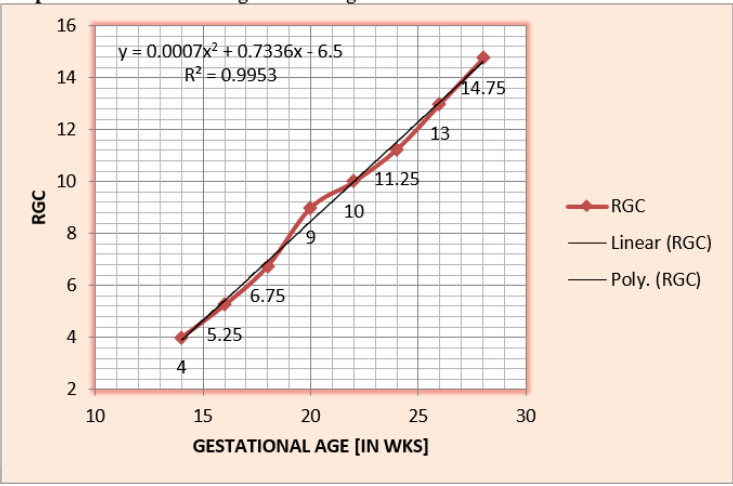

Graph 2 shows that from 14 to 28 weeks, fetus shows active glomerulogenesis. So RGC increases with increase in gestational age. Correlation coefficient value $\left[R^{2}=0.99\right]$ suggests significant linear correlation of RGC with mid gestational age.

\section{Graph 3: Correlation of average kidney weight and} RGC

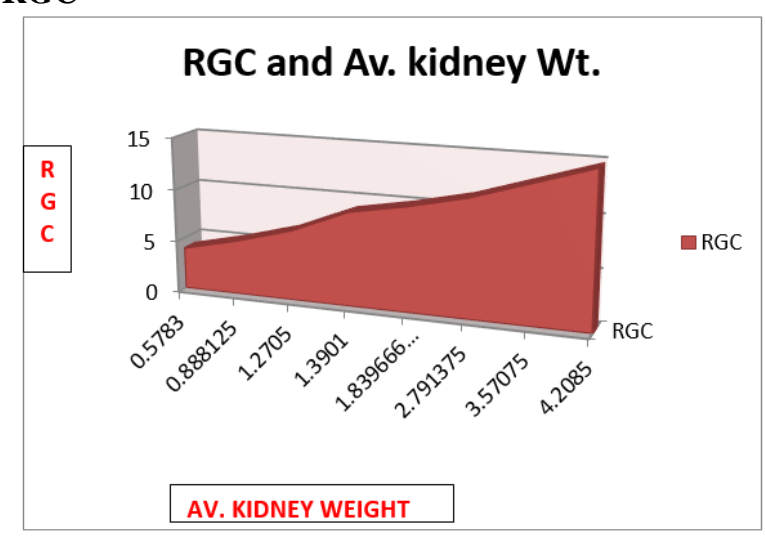

Graph 3 suggests that as the fetal kidney weight increases, number of glomerular generation also increases proportionally. Most active glomerulogenesis 
[+2.25of RGC] is seen between 18 to 20 weeks of gestational age.

\section{Discussion}

RGC calculated by counting the number of glomeruli formed along a medullary ray from the corticomedullary junction to the outer renal cortex. This method has been validated by Hinchliffe and colleagues. ${ }^{5}$

In circumstances when clear medullary rays were not observed, a straight line was drawn from the corticomedullary junction to the outer renal cortex and all mature glomeruli along the line were counted according to the protocol of Rodriguez and colleagues. ${ }^{6}$

The present study follows protocol of both the workers to calculate RGC.

Shimda K. ${ }^{3}$ observed that "the RGC increased steadily with gestational weeks, and gradually reached a plateau of around 12 counts at about 36 weeks. It was not influenced by the intrauterine fetal growth".

Tsuda $^{4}$ (1943) stated that" the first glomerulus appears when the embryo is 3 inches long. At 4-5 months [13- 20 weeks], he found 3-5 layers of glomeruli; at 7-9 months (25 -36 weeks) 9-12 layers, and at the 10th month (37- 40 weeks) 10-15 layers."

Kumar and Pillay ${ }^{7}$ and Piercecchi-Marti et al. ${ }^{8}$ in their respective studies states the definite positive correlation between fetal age and number of glomerular generations.

In the present study active glomerulogenesis continued from 14 to 28 weeks of age. Therefore the average RGC is gradually increasing from 4 to 15 counts in mid gestational age with the maximum phase of growth [+2.25] in $18-20$ weeks. It supports the results of the above workers.

Chikkannaiah P, Roy et $\mathrm{al}^{9}$ was calculated that "for every increase by one glomerular generation, there will be an increase in the period of gestation by approximately 2.5 weeks".

The present study noted that with increase in one glomerular generation, there will be increase in 1.30 weeks of gestational age. It suggests that the RGC can express human fetal kidney growth as active glomerulogenesis in intrauterine life.

Sutherland MR et $\mathrm{al}^{10}$ noted that there was a significant positive correlation between kidney weight and the number of glomerular generations. Present study supports the above results.

\section{Conclusion}

The present study concluded that RGC is highly sensitive parameter for assessment of nephrogenesis. The histomorphologic determination of RGC can be utilised as a reliable alternative method of estimating gestational age. It is a sensitive parameter for detection of both hypoplasia and maturation delay in relation with gestational age and renal weight. The observed growth tendencies of fetal glomerulus in human fetal life by RGC could be useful to assess the kidney maturity through invasive or noninvasive investigative methods in the future.

\section{References}

1. Rodríguez MM, Gómez AH, Abitbol CL, Chandar JJ, Duara S, Zilleruelo .Histomorphometric analysis of postnatal glomerulogenesis in extremely preterm infants. Pediatr. Dev. Pathol. 2004;7:17-25.

2. Rosenblum ND. Developmental biology of the human kidney. Semin Fetal Neonatal. Med. 2008;13(3):125-32.

3. Shimda K., Hosokawa S., Tohda A. Histological study of fetal kidneys. Development of nephrons according to gestational weeks. Nihhon Hinyokika Gakkai Zasshi. 1993;84(12):2091-6.

4. Tsuda. Histologic investigation of fetal kidney. Jap J.Obst.Gynec.1943;17:227-341.

5. Hinchliffe SA, Sargent PH, Chan YF, van Velzen D, Howard CV, Hutton JL, Rushton DI. "Medullary ray glomerular counting" as a method of assessment of human nephrogenesis.

6. Rodriguez MM, Gomez AH, Abitbol CL, Chandar JJ, Duara S, Zilleruelo GE. Histomorphometric analysis of postnatal glomerulogenesis in extremely preterm infants. Pediatr Dev Pathol , 2004;7:17-25.

7. Kumar KU, Pillay VV. Estimation of fetal age by histological study of kidney. Med Sci Law 1996;36:22630.

8. Piercecchi-Marti M, Adalian P, Liprandi A, FigarellaBranger D, Dutour O, Leonetti G. Fetal visceral maturation: A useful contribution to gestational age estimation in human fetuses. J Forensic Sci 2004;49:1-6.

9. Chikkannaiah P, Roy M, Kangle R, Patil PV. Glomerulogenesis: Can it predict the gestational age? A study of 176 fetuses. Indian J Pathol Microbiol 2012;55:303-7

10. Megan R. Sutherland, Lina Gubhaju, Lynette Moore, Alison L. Kent. Accelerated Maturation and Abnormal Morphology in the Preterm Neonatal Kidney, J Am Soc Nephrol. Jul 2011;22(7):1365-74. 